\title{
Occupancy Detection and People Counting Using WiFi Passive Radar
}

\author{
Chong Tang*, Wenda $\mathrm{Li}^{*}$, Kevin Chetty*, Simon Julier ${ }^{\ddagger}$, Karl Woodbridge ${ }^{\dagger}$ \\ *Department of Security and Crime Science, University College London, UK \\ $\dagger$ Department of Electronic and Electrical Engineering, University College London, UK \\ $\ddagger$ Department of Computer Science, University College London, UK \\ * \{chong.tang.18, wenda.li, k.chetty, k.woodbridge, s.julier\}@ucl.ac.uk
}

\begin{abstract}
Occupancy detection and people counting technologies have important uses in many scenarios ranging from management of human resources, optimising energy use in intelligent buildings and improving public services in future smart cities. Wi-Fi based sensing approaches for these applications have attracted significant attention in recent years because of their ubiquitous nature, and ability to preserve the privacy of individuals being counted. In this paper, We present a Passive WiFi Radar (PWR) technique for occupancy detection and people counting. Unlike systems which exploit the Wi-Fi Received Signal Strength (RSS) and Channel State Information (CSI), PWR systems can directly leverage any environment covered by an existing WiFi local area network without special modifications to the Wi-Fi access point. Specifically, we apply Cross Ambiguity Function (CAF) processing to generate Doppler spectrograms, and employ a CLEAN algorithm to remove the direct signal interference. A Convolutional Neural Network (CNN) and sliding-window based feature selection scheme is then used for classification. Experimental results collected from a typical office environment have validated that the proposed PWR system is able to determine room occupancy with $99.5 \%$ accuracy, and correctly count $(\mathbf{9 8 . 1 \%})$ how many people are present when there are up to four people.
\end{abstract}

Index Terms-WiFi Sensing, Occupancy Detection, Crowd Counting, Passive WiFi Radar, CNN

\section{INTRODUCTION}

Occupancy detection and people counting are widely required in many scenarios. For example, tracking queuing and sitting situations can improve service quantity by rearranging staff resources in shopping malls or restaurants. In terms of optimizing energy use, detecting occupancy state can reduce unnecessary lighting or/and heating cost in an intelligent building. In public transportation and for safety concern, estimating the number of passengers at a bus stop or train platform can make management center adjust schedule to avoid overcrowding situations. In some security aspects, for instance, occupancy detection can make sure no one has entered an area once it has been checked for explosives. Some InfraRed and camera-based detectors have been employed in many application areas, but with large detection errors and privacy exposure issues, their reliability and performance are limited. On the contrary, recent WiFi-based system has many unique advantages to avoid these problems. It directly leverages $\mathrm{WiFi}$ signal which ubiquitously exists in surrounding environments and has ability to penetrate through objects. As a result, WiFibased systems can guarantee large monitoring areas and be less limited by lighting or light-of-sight conditions, whilst also preserving privacy.

Common WiFi sensing researches mainly focus on RSS and/or CSI data. RSS data is an index of the WiFi energy. With the increase of the distance from WiFi AP, the value of RSS decreases. Based on this feature, RSS-based systems can be used for localization[1] and gesture recognition[2][3]. For occupancy sensing, many studies has proposed. For example, in[4], they estimate the number of passengers on a bus by deploying four WiFi sensors to collect RSS data from carried mobile devices. Researchers in [5] propose a device-free RSSbased system which uses existing WiFi networks and applies classifiers to estimate the number of people. They report 98.20\% occupancy state detection accuracy but this drops to $77.20 \%$ when attempting to count the number of people in the crowd. However, because of the presence of multiple reflection and scattering paths, it cannot distinguish them and may appear unpredictable fluctuations which makes detection suffer from large false positive. In this case, RSS-based system often requires intensive offline training and densely deploys WiFi communication links which reduces simplicity and convenience of the system. On the other hand, for CSI-based system, it is an upgrade version of RSS which describes each component in multi-path propagation with the format of CSI for a clearer understanding of propagation information. As an Orthogonal Frequency Division Multiplexing(OFDM)-based transmission, WiFi signals are encoded with a set of orthogonal subcarriers with corresponding CSI values. The system can gather raw CSI values via Network Interface card(NIC). For example, in [6], using a modified firmware of the IWL 5300 can collect CSI values for 30 OFDM subcarriers. This superiority made CSI-based system become one of the most popular WiFi sensing techniques. Studies ave shown its ability to detect bulk human motions such as walking and sitting[7], as well as slight movements such as breathing[8], heartbeats[9] and lip movements. [10][11][12][13] have proposed some CSIbased device-free crowd counting systems. Nevertheless, most of CSI-based systems require a laptop or PC with a modified WiFi NIC to act as a receiver. Meanwhile, some CSI-based systems require manipulation of WiFi APs to transmit data-rate 
signal in order to make use of it's full allowable bandwidth, which has real-world implications in terms of how it may affect the throughput for the users of the communication network. For implementing occupancy detection system in all kinds of scenarios and reducing system complexity, we need an effective strategy leveraging existing WiFi infrastructure without any additional modifications, as well as maintaining even improving detection accuracy.

Recent researches in radio-frequency(RF) sensing community has shown significant interest in PWR system because of low cost and ability to covertly detect and track noncooperative targets. Many PWR applications have been proposed for example, through-the-wall[14] and indoor sensing scenarios. Different from RSS and CSI-based systems, PWR system extract target Doppler information through CAF processing. Doppler spectrogram can present meaningful Doppler information for better visualization and understand the data. Furthermore, PWR-based system does not require extra modification and can directly use existing WiFi network and still has the ability to detect individuals in high-clutter indoor environments [REFERENCE- CHETTY09] and through walls [14]. It is also highly sensitive to small motions due to the long integration times possible and has even been shown to detect the perturbations of the chest-wall during breathing [15]. However, to the best of our knowledge no work has been spent to investigate PWR system in the occupancy detection and people counting. This paper presents our first attempt of applying PWR system in this important area. Specifically, we used a CLEAN algorithm to filtering undesired signals to enhance the detection performance. Afterwards, Convolutional Neural Networks (CNNs) is adopted to learn a general model from Doppler spectrogram. Instead of directly feeding CAFs into CNNs, we employed sliding-window to select features from Doppler spectrogram which highly improves estimation accuracy. With these techniques, in this paper, we demonstrate its ability to outperform aforementioned systems for occupancy sensing and people counting purpose.

In summary, we mainly make the following contributions:

- To the best of author's knowledge, we are the first to apply PWR system and use Doppler spectrogram data for occupancy detection and people counting purposes.

- We proposed new feature selection scheme rather than directly training a single CAF to increase prediction accuracy.

- Experimental results have validated that PWR system is able to achieve maximum $99.53 \%$ occupancy detection accuracy and $98.14 \%$ crowd counting accuracy(up to 4 people).

The rest of the paper is organized as follows: Section II describes the fundamental mechanism of PWR system. Next, Section III introduces details of experiments including equipment setup, experiment design and data collection. Section IV presents experimental results and evaluates the performance of different CNN models. Finally, We conclude this paper and discuss possible extension for future work in Section V.

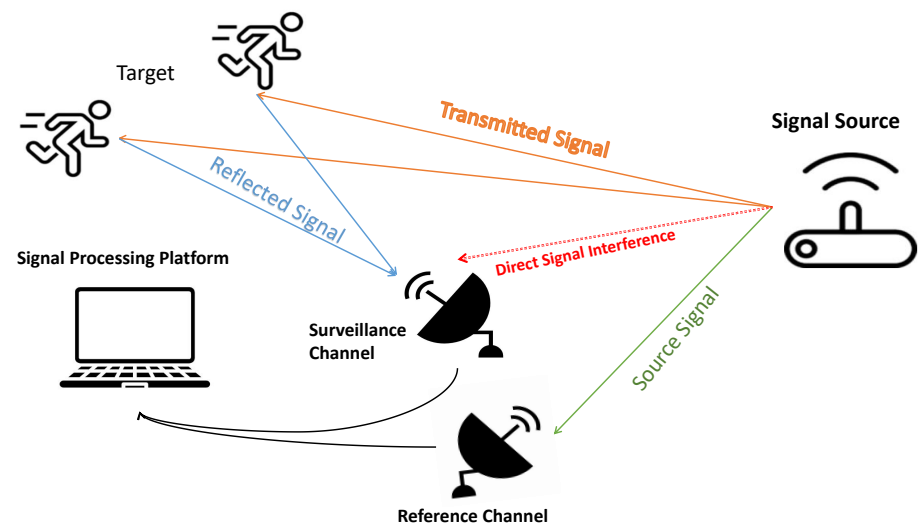

Fig. 1: The common layout for a passive radar system

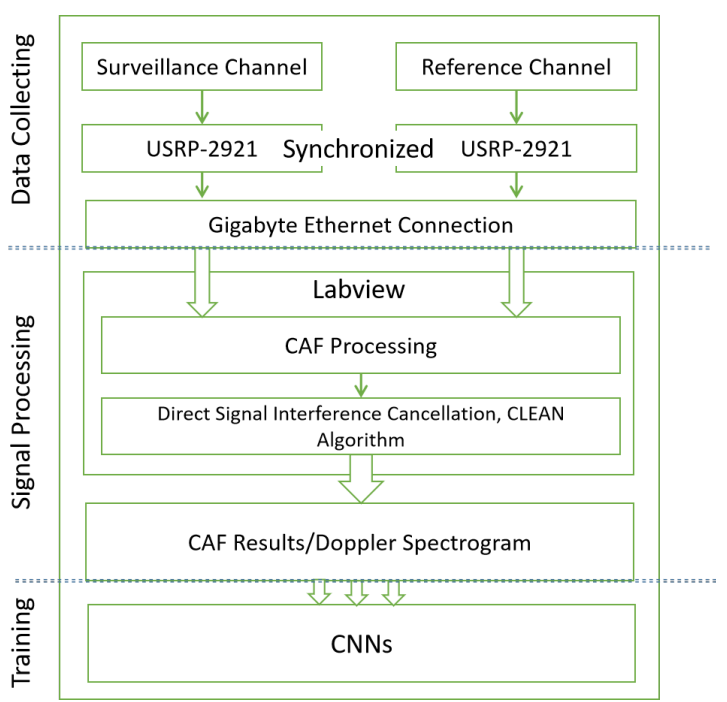

Fig. 2: Block Diagram of System

\section{Passive WiFi Radar SYSTEM}

\section{A. System Architecture}

Passive Radar is a kind of bi-static radar system which uses the RF signal emitted by existing signal sources like TV signals to achieve detection. Recent applications include tracking airborne targets, monitoring traffic and sensing human activities. The passive radar system uses separately located antennas, named surveillance channel and reference channel respectively, and cross-correlation techniques to measure variations between reflected signal from the target and direct signal from the signal source. It should be noted that two channels need to be synchronized. Thereby, the corresponding Doppler strength and range information from the RF source via the target to the surveillance antenna is determined. A sketch of passive radar system setup has shown in Fig. 1. Specially, PWR system is a passive radar system using WiFi signals. The general concepts are similar with others but with slight difference because of the characteristic of WiFi signals. Bandwidth of commercial WiFi signal is fixed at 20 to $40 \mathrm{MHz}$ corresponding to 7.5 to 15 meters range resolution, which is 
not enough for indoor environments. As a result, proposed system focuses on analyzing Doppler strength of CAF results or Doppler spectrogram. Fig. 2 has shown the block diagram of proposed system.

\section{B. Signal Processing for PWR System}

In passive radar, signals from surveillance channel and time-delayed copies of reference signals are cross-correlated by $\mathrm{CAF}$ to extract range and Doppler information. A CAF mapping equation can be defined as equation (1):

$$
C A F\left(\tau, f_{d}\right)=\int_{0}^{T} S_{\text {sur }}(t) S_{\text {ref }}^{*}(t-\tau) e^{j 2 \pi f_{d} f_{c} t} d t
$$

where $S_{\text {sur }}(t)$ represents received signal from surveillance channel, $S_{\text {ref }}(t-\tau)$ is replicas of time-delayed reference signals and $\tau$ is the delay, $f_{d}$ is the Doppler shift and $f_{c}$ represents the carrier frequency, $[*]$ operator denotes the complex conjugate. Meanwhile, integration time $T$ determines how long period of signals will be processed. In IEEE 802.11 standard, WiFi signal propagation is modulated by OFDM scheme[16], so we can define pure reference signals $\left(S_{r e f}\right)$ as equation (2):

$$
S_{\text {ref }}(t)=\frac{1}{\sqrt{N}} \sum_{n=0}^{N-1} a_{n} e^{j 2 \pi n t}
$$

where $\mathrm{N}$ is the number of OFDM symbols for each carrier $a_{n}, n$ is the index of a symbol. On the other hand, WiFi signal propagation has multiple path caused by reflections from stationary objects or moving people. Compared with their source signal, they might be attenuated, delayed or have phase shift. In this case, these signals i.e. $S_{\text {sur }}$ can be received and described in the form of equation (3):

$$
S_{\text {sur }}(t)=\sum_{p} A_{p} e^{j 2 \pi f_{d} f_{c} t} x(t-\tau)+n(t)
$$

where $\mathrm{p}$ represents the number of transmission paths, $A_{p}$ is the attenuation factor, and $n(t)$ represents the Additive White Gaussian Noise(AWGN). Substitute equation (2) and (3) into (1), CAF values involving range and Doppler information can be obtained. However, the above processing does not account impact of noise and there is still some interference which needs extra processing operations. One of the major interference is the strong signals directly coming from WiFi AP. Because of much less attenuation, it will shade other reflected signals and cause unwanted peaks in the zero Doppler bin. The CLEAN algorithm can remove this direct signal interference by selfcancellation, which can be described as (4):

$$
C A F^{\prime}\left(\tau, f_{d}\right)=C A F\left(\tau, f_{d}\right)-\alpha C A F_{\text {self }}\left(\tau-T_{k}, f_{d}\right)
$$
where $C A F_{\text {self }}\left(\tau, f_{d}\right)$ represents the CAF over the reference channel, $\alpha$ is the maximum absolute value of $C A F\left(\tau, f_{d}\right)$. Then, after employing CLEAN algorithm, desired Doppler information is uncovered.

\section{Monitoring occupancy information with Doppler spectro- gram}

PWR system has ability to detect small movements like breathing. Unlike Infra-Red sensor, as long as there is one person in the monitoring area, Doppler power will not be

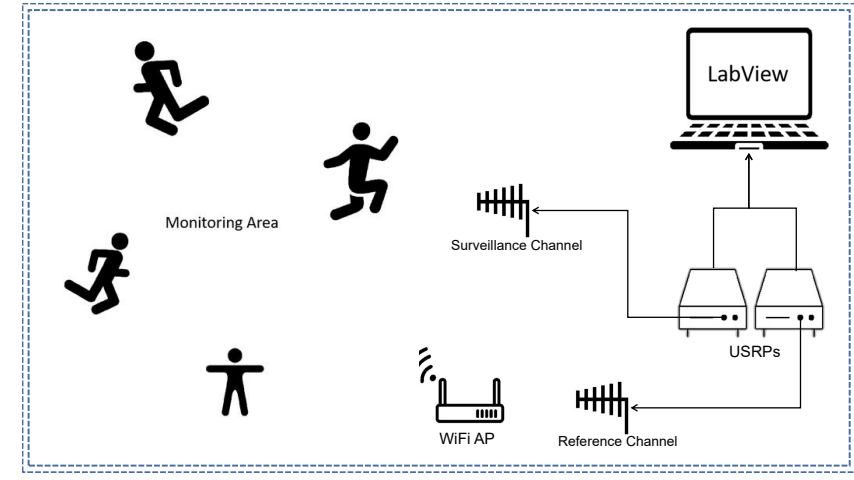

Fig. 3: An example layout in experimental environment

zero. According to this fact, it is easy to determine occupancy state via analyzing whether Doppler power is zero on CAF. Nevertheless, for crowd counting task, it becomes much more complex than previous one. As mentioned before, range resolution of PWR detection is around 7.5 to 15 meters, for around 5 meters by 5 meters experimental room, it is not able to distinguish locations of different people which may cause underestimation problem. To solve it, the idea is that although locations of people are unknown, the Doppler power and density will be varied with changes of the number of people. For example, one person cannot move towards two directions at the same time, therefore, if Doppler spectrogram shows non-zero Doppler power at both directions, it means there are at least two people in the monitoring area. On the other hand, the strength of Doppler power for different activities for one person has a certain range, moreover, the range is normally quite limited because we do not do fierce movements very often. As a result, if Doppler power of one bin exceeds maximum value of the range, it actually indicates that more than one person existing in the monitoring area. Meanwhile, human activities have temporal relations. For example, we can observe a clear sinusoidal waveform while one person is breathing. Hence, if such the waveform can be recognized, it will be an index for detection.

Based on one of these characteristics or combination of them, PWR system can achieve both occupancy state detection and crowd counting. But due to random movements of people, patterns of Doppler spectrogram are still hard to extract by observing or specific mathematical model, we introduced CNN to automatically learn a general model from dataset.

\section{SySTEM IMPLEMENTATION}

In this section, we present the structure of PWR system in experimental scenarios including required equipment and example layout. Also, details about experiments and dataset are introduced in below.

\section{A. System Overview}

Fig. 3 shows example system layout in our experimental environment. There are one Yagi antenna for reference channel to capture source signals from WiFi AP and one Yagi antenna 
for surveillance channel to monitor region of interest. In addition, two NI USRP-2921 for real-time signals acquisition are connected with two antennas through a Gigabyte Ethernet, respectively. Then measured data is delivered to computational unit which is based on LabVIEW for signal processing steps. Moreover, a camera system operated by Raspberry Pi has been deployed, and the video data can be used as the ground truth of PWR occupancy sensing experiments. Meanwhile, the camera system has been synchronized with PWR system through an NTP server.

\section{B. Experiments Design}

We designed a 45 minutes continuous experiment to verify the feasibility of PWR system for occupancy sensing. The whole measurement has four participants and includes nine sub-experiments (lasting five minutes for each one). Both camera and PWR systems kept working during the experiment. The initial state in monitoring area was non-occupied state i.e. zero people. After the first five minute passed, four participants successively entered into the monitoring area and kept randomly walking to simulate a real scenario like people are walking in a train station. Thereby, the occupancy state in the monitoring area varied from non-occupied state to occupied state and the number of people increased from one person to maximum four people. Then the number of people continues to change several times to get more data. Finally, the number of participants in nine sub-experiments are in order of $0,1,2,3,4,3,2,1$ and 4 .

\section{Dataset Explanation and CNNs}

The measuring rate of PWR system is $10 \mathrm{CAFs}$, as known as 10 frames, per second. At the end, we collected 45 minutes data resulting in around 27,000 frames, which are labelled according to recorded video from the camera system. There are two labelling schemes corresponding to different purposes. For occupancy state detection, we labelled 'non-occupied' and 'occupied' for zero people state and the rest of states, respectively. For crowd counting, we labelled data according to the number of people such as 'one person', 'two people', etc. Meanwhile, the CAF strength is normalized within $[0,1]$ at the beginning. This is not only for faster gradient descent in deep neural network, but also for reducing impacts of varied WiFi signals and different monitoring areas.

One the other hand, as discussed in Section III.C, due to spatial-temporal relation of movements of multiple people, rather than learning from one single $\mathrm{CAF}$, combining continuous frames as one feature, could highly improve estimation accuracy. To verify this guess, we used different sizes of sliding-window to extract features from spectrogram and trained them. The width of sliding-window was set to 5, 7 and 10 for each training where we also trained single CAF data for comparison. Basically, with the increase of window size, richer information is presented on the Doppler spectrogram. Besides, the label of a feature is determined by labels of each frames in the window. For simplicity, we will make sure that

\begin{tabular}{|l|l|l|l|l|l|}
\hline Parameters & $\begin{array}{l}\text { Non- } \\
\text { occupied }\end{array}$ & $\begin{array}{l}\text { One per- } \\
\text { son }\end{array}$ & $\begin{array}{l}\text { Two } \\
\text { people }\end{array}$ & $\begin{array}{l}\text { Three } \\
\text { people }\end{array}$ & $\begin{array}{l}\text { Four } \\
\text { people }\end{array}$ \\
\hline Mean & 0.002 & 0.005 & 0.011 & 0.013 & 0.014 \\
\hline Median & 0.000 & 0.0017 & 0.002 & 0.003 & 0.004 \\
\hline std & 0.005 & 0.018 & 0.028 & 0.029 & 0.032 \\
\hline
\end{tabular}

TABLE I: Mean, Median and Standard deviation values for different occupancy states

there is only one label type existing in the window. Features including mixed labels will be not fed into CNNs.

In aspect of deep neural network, we selected CNNs techniques to train the dataset and leveraged 5-folds crossvalidation method to evaluate models. To determine which structures more fit to our case, we employed three popular architectures including LeNet[17], AlexNet[18] and ZFNet[19] and slightly modified them according to the demand. The biggest modification is that we reduced the number of pooling layers. That is because each pixels on Doppler spectrogram involves the strength and direction information, but pooling layers may weaken information of some pixels or make them lost which is detrimental for the estimation.

\section{Experimental and Neural Network Results}

Fig. 4 presents fragments of Doppler spectrogram for different occupancy states. Furthermore, Fig. 5 presents examples of CAF plots for different states. What needs illustration is that the Doppler spectrogram has 100 Doppler bins in one frame/column where bins above the middle line represent positive movement direction while bins below the middle line is negative direction. Here, positive and negative directions refer to moving towards and away surveillance antenna, respectively. Furthermore, the color bars have shown the level of Doppler power. On the other hand, Table I statistically shows some parameters for different states. Finally, Table II list results of CNNs.

In Fig. 4, the spectrogram of empty room (a) indicates low-amplitude $(<0.01)$ noises still exist but will not make much difference for results. For spectrogram of one person (b), we can clearly see a sinusoid-like wave which represents continuous forward and backward walking. But with the increase of the number of people (Fig. 4(c)(d)(e)), the strength and density of Doppler power increased resulting in the entire Doppler spectrogram also becomes more and more complex.

One the other hand, in Fig. 5, the CAF of empty room still has slight noises but with much less amplitude than other results of occupied states. Furthermore, different from CAFs in Fig. 5(c)(d)(e), the CAF of one person only has strength in one direction which is consistent with analysis in Section III.C.

In TableI, we calculated mean, median and standard deviation parameters for different people states. As can be seen, differences between each states accord with changes on Doppler spectrogram. On the other hand, results of CNNs have been listed in TableII, whilst example confusion matrices of ZFNet results with different window sizes is shown in Fig. 6. TableII counts estimation accuracy of both occupancy state detection 

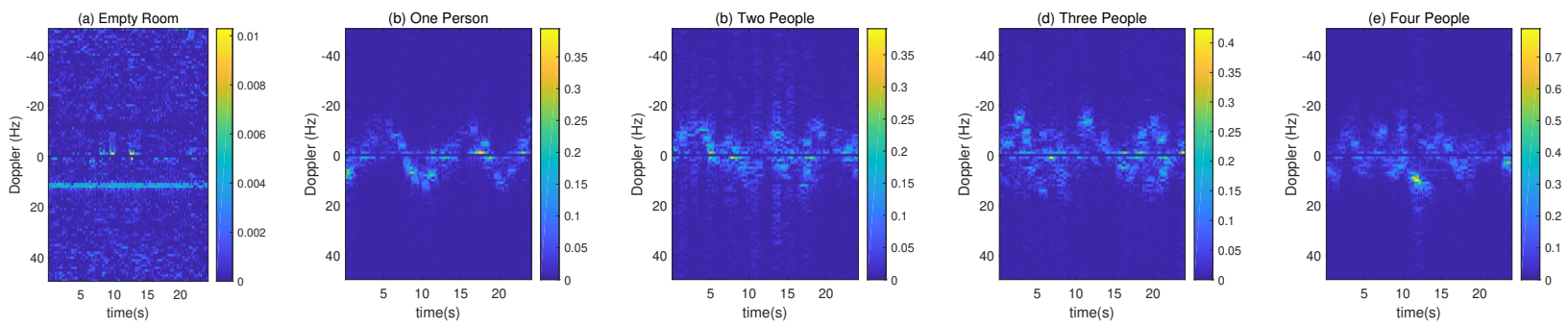

Fig. 4: Doppler spectrogram fragment of different occupancy states
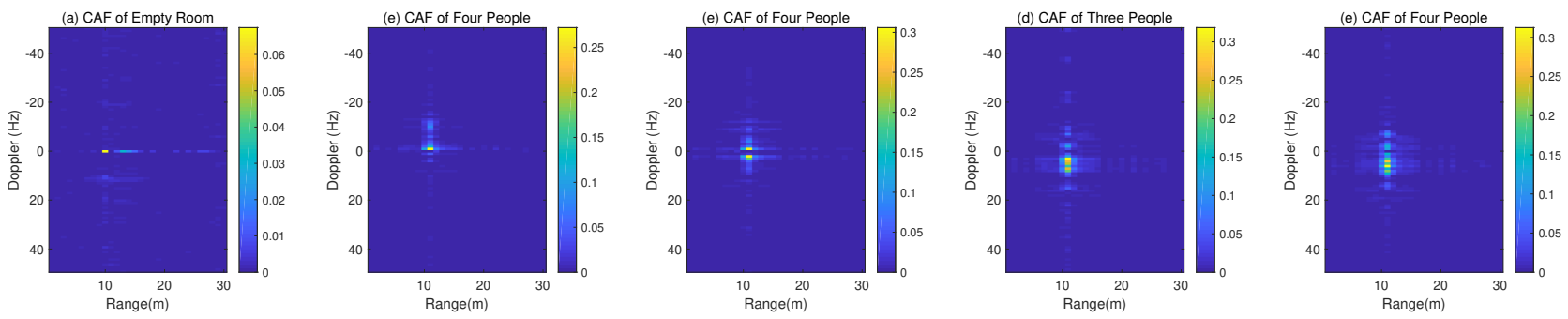

Fig. 5: CAF results of different occupancy states

\begin{tabular}{|c|c|c|c|}
\hline Neural Network & Window Size & Classes & Accuracy $(\%)$ \\
\hline \multirow{8}{*}{ LeNet } & \multirow{2}{*}{ CAF } & occupancy sensing & 91.5 \\
\hline & & people counting & 83.4 \\
\hline & \multirow{2}{*}{5} & occupancy sensing & 96.6 \\
\hline & & people counting & 95.6 \\
\hline & \multirow{2}{*}{7} & occupancy sensing & 98.2 \\
\hline & & people counting & 96.9 \\
\hline & \multirow{2}{*}{10} & occupancy sensing & 99.5 \\
\hline & & people counting & 98.8 \\
\hline \multirow{8}{*}{ AlexNet } & \multirow{2}{*}{ CAF } & occupancy sensing & 91.2 \\
\hline & & people counting & 84.1 \\
\hline & \multirow{2}{*}{5} & occupancy sensing & 96.9 \\
\hline & & people counting & 93.2 \\
\hline & \multirow{2}{*}{7} & occupancy sensing & 97.23 \\
\hline & & people counting & 96.1 \\
\hline & \multirow{2}{*}{10} & occupancy sensing & 98.6 \\
\hline & & people counting & 98.3 \\
\hline \multirow{8}{*}{ ZFNet } & \multirow{2}{*}{ CAF } & occupancy sensing & 92.4 \\
\hline & & people counting & 85.2 \\
\hline & \multirow{2}{*}{5} & occupancy sensing & 95.9 \\
\hline & & people counting & 94.3 \\
\hline & \multirow{2}{*}{7} & occupancy sensing & 96.4 \\
\hline & & people counting & 95.3 \\
\hline & \multirow{2}{*}{10} & occupancy sensing & 98.7 \\
\hline & & people counting & 97.3 \\
\hline
\end{tabular}

TABLE II: Experimental Results

and people counting. We can find that with the increase of window size, estimation accuracy highly improved, especially from using a single CAF (i.e. window size $=1$ ) to using a short period of Doppler spectrogram (i.e. window size $i 1$ ). Overall, PWR occupancy sensing system can finally achieve maximum $99.54 \%$ accuracy for occupancy state detection task (using the modified LeNet with window size equals 10) and maximum $98.14 \%$ accuracy for crowd counting task (using the modified ZFNet with window size equals 10).

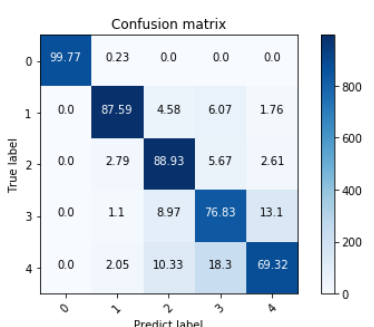

(a) window size is 1 i.e. $\mathrm{CAF}$

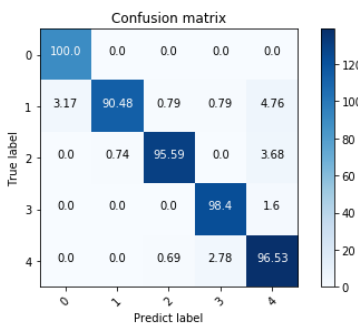

(c) window size is 7

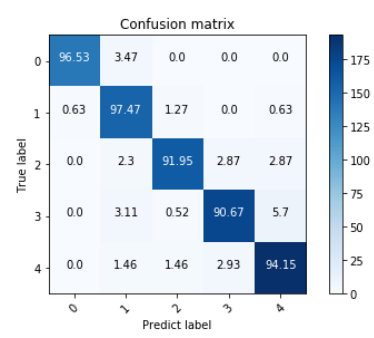

(b) window size is 5

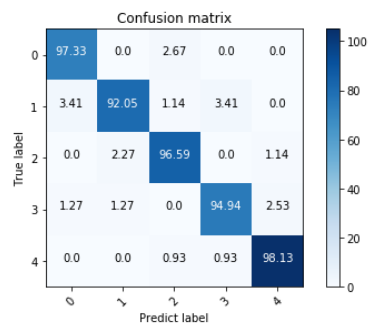

(d) window size is 10
Fig. 6: Confusion matrix ZFNet with different window sizes, where labels represents the number of people

\section{CONCLUSiOn AND Future Work}

In this paper, we demonstrate that a PWR system is able to achieve occupancy state detection and crowd counting. Different from other WiFi sensing systems, the PWR system does not require any extra modifications or devices like NIC on WiFi networks and can directly leverage any commercial WiFi AP for detection. Moreover, instead of measuring RSS and CSI data, a PWR system cross-correlates received signals from two channels with CAF method and removes the strong 
direct signal with CLEAN algorithm to generate time-varied Doppler spectrogram. Compared with RSS and CSI data, the Doppler spectrogram is more meaningful in which we can roughly observe waveform of movements. Finally, by training with CNNs, we obtain general models for occupancy sensing which can achieve quite good estimation accuracy. Besides, we also have summarized some aspects needed to be noticed for training Doppler spectrogram data including applying sliding-window method with relative bigger window size, more complex $\mathrm{CNN}$ and reducing pooling layers in the earlier layers. In the end, it is envisioned that a PWR occupancy sensing system can be extensively applied in many scenarios because of its low-cost, simplicity and convenience, more importantly having quite high detection accuracy.

For our future's plan, because this paper mainly focused on exploring the feasibility of using PWR system for occupancy sensing, the number of participants and the diversity of experimental scenarios are not so much. In the future, we are going to carry out more experiments with more participants in various environments so that we can further verify the reliability of the system. We believe that based on the current results, PWR system will perform well in future's experiments as well.

\section{REFERENCES}

[1] D. Park and J. G. Park, "An enhanced ranging scheme using wifi rssi measurements for ubiquitous location," in 2011 First ACIS/JNU International Conference on Computers, Networks, Systems and Industrial Engineering. IEEE, 2011, pp. 296-301.

[2] S. Sigg, S. Shi, F. Buesching, Y. Ji, and L. Wolf, "Leveraging rf-channel fluctuation for activity recognition: Active and passive systems, continuous and rssi-based signal features," in Proceedings of International Conference on Advances in Mobile Computing \& Multimedia, 2013, pp. 43-52.

[3] S. Sigg, M. Scholz, S. Shi, Y. Ji, and M. Beigl, "Rf-sensing of activities from non-cooperative subjects in device-free recognition systems using ambient and local signals," IEEE Transactions on Mobile Computing, vol. 13, no. 4, pp. 907-920, 2013.

[4] U. Mehmood, I. Moser, P. P. Jayaraman, and A. Banerjee, "Occupancy estimation using wifi: A case study for counting passengers on busses," in 2019 IEEE 5th World Forum on Internet of Things (WF-IoT). IEEE, 2019, pp. 165-170.

[5] T. Yoshida and Y. Taniguchi, "Estimating the number of people using existing wifi access point in indoor environment," in Proceedings of the 6th European Conference of Computer Science (ECCS'15), 2015, pp. 46-53.

[6] S. Di Domenico, M. De Sanctis, E. Cianca, and G. Bianchi, "A trained-once crowd counting method using differential wifi channel state information," in Proceedings of the 3rd International on Workshop on Physical Analytics, 2016, pp. 37-42.

[7] W. Wang, A. X. Liu, M. Shahzad, K. Ling, and S. Lu, "Devicefree human activity recognition using commercial wifi devices," IEEE Journal on Selected Areas in Communications, vol. 35, no. 5, pp. 11181131, 2017.

[8] H. Wang, D. Zhang, J. Ma, Y. Wang, Y. Wang, D. Wu, T. Gu, and B. Xie, "Human respiration detection with commodity wifi devices: do user location and body orientation matter?" in Proceedings of the 2016 ACM International Joint Conference on Pervasive and Ubiquitous Computing, 2016, pp. 25-36.

[9] X. Wang, C. Yang, and S. Mao, "Phasebeat: Exploiting csi phase data for vital sign monitoring with commodity wifi devices," in 2017 IEEE 37th International Conference on Distributed Computing Systems (ICDCS). IEEE, 2017, pp. 1230-1239.

[10] S. Depatla and Y. Mostofi, "Crowd counting through walls using wifi," in 2018 IEEE International Conference on Pervasive Computing and Communications (PerCom). IEEE, 2018, pp. 1-10.
[11] S. Kianoush, S. Savazzi, and M. Nicoli, "Device-free crowd sensing in dense wifi mimo networks: Channel features and machine learning tools," in 2018 15th Workshop on Positioning, Navigation and Communications (WPNC). IEEE, 2018, pp. 1-6.

[12] W. Xi, J. Zhao, X.-Y. Li, K. Zhao, S. Tang, X. Liu, and Z. Jiang, "Electronic frog eye: Counting crowd using wifi," in IEEE INFOCOM 2014-IEEE Conference on Computer Communications. IEEE, 2014, pp. 361-369.

[13] M. De Sanctis, T. Rossi, S. Di Domenico, E. Cianca, G. Ligresti, and M. Ruggieri, "Lte signals for device-free crowd density estimation through csi secant set and svd," IEEE Access, vol. 7, pp. 159943$159951,2019$.

[14] K. Chetty, G. E. Smith, and K. Woodbridge, "Through-the-wall sensing of personnel using passive bistatic wifi radar at standoff distances," IEEE Transactions on Geoscience and Remote Sensing, vol. 50, no. 4, pp. 1218-1226, 2011.

[15] W. Li, B. Tan, and R. J. Piechocki, "Non-contact breathing detection using passive radar," in 2016 IEEE International Conference on Communications (ICC). IEEE, 2016, pp. 1-6.

[16] P. Falcone, F. Colone, C. Bongioanni, and P. Lombardo, "Experimental results for ofdm wifi-based passive bistatic radar," in 2010 ieee radar conference. IEEE, 2010, pp. 516-521.

[17] Y. LeCun, L. Bottou, Y. Bengio, and P. Haffner, "Gradient-based learning applied to document recognition," Proceedings of the IEEE, vol. 86, no. 11, pp. 2278-2324, 1998.

[18] A. Krizhevsky, I. Sutskever, and G. E. Hinton, "Imagenet classification with deep convolutional neural networks," in Advances in neural information processing systems, 2012, pp. 1097-1105.

[19] M. D. Zeiler and R. Fergus, "Visualizing and understanding convolutional networks," in European conference on computer vision. Springer, 2014, pp. 818-833. 